\title{
Learning, Unlearning, and Relearning Issues of Anatomy Cadaver Management during COVID-19 Pandemic in India and World: Thoughts for Present and Probable Future Trends
}

Nidhi Gupta ${ }^{1}$, S. Pandey ${ }^{2}$

\section{ABSTRACT}

The current scenario of Pandemic has disrupted the classic morbid anatomy teaching dissection module for both educators and trainee. Moreover, lack of universal system of procedure for cadaver management optimization in context of education purpose is missing and not optimized. Debates for advocacy or avoidance of cadaveric dissection with probable COVID-19 infection, so far had been inconclusive. Pragmatic guidelines for cadaver reception for scholastic purpose, safe disposal, best forensic practice concerned with health personnel staff, technical and ethical issues are upcoming at a brisk pace although arbitrary implying standardization. There is a need for finding coherence and congruence with directives of regulatory bodies for medical teaching and available acts in current scenario. Hence, we endeavor for providing an insight into the needs and solutions in light of recent evidences.

KEY WORDS: Anatomy dissection, cadaver management, embalming.

\section{Evidence based insights for Anatomy cadaver} management in COVID-19 Pandemic

Disruptions in morbid anatomy dissection modules in medical institutes due to current pandemic have left the perplexed educators at crossroads of dilemma. Anatomists worldwide at present have no universal system of procedure for educational purpose utilized cadaver management. To cite, two cadavers having died of coronavirus pneumonia, donated after approval of relatives, and were dissected at Wuhan Jinyintan Hospital. ${ }^{[1]}$ The observers strongly advocated early dissection and expressed serious disappointments for being late regarding noting

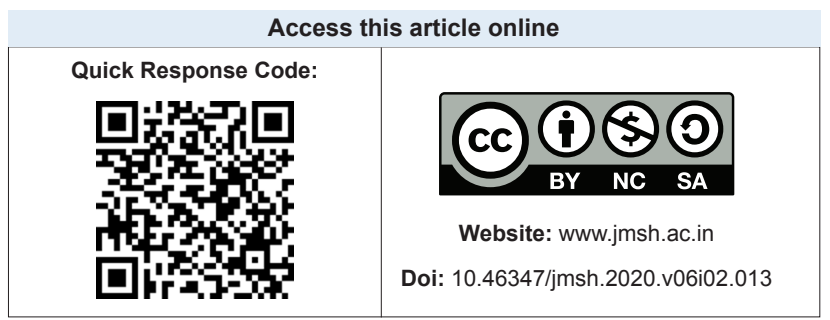

important morbid/histopathological findings. ${ }^{[1]}$ Instead of safe disposal, call for more donors within specified laws and regulation was made. ${ }^{[1]}$ To confuse further, authors claiming worldwide first fatal case of novel coronavirus spreading from a corpse to a medical examiner in Thailand produced corrigendum for misinterpretation later. ${ }^{[2]}$ World over the dilemma continues regarding working within assigned framework of cadaver management for scholastic purpose. At present, the balance between need of hour and technical and ethical issue is quite arbitrary implying urgent need of national and international standard guidelines. International Committee of the Red Cross (ICRC) since 2000 is working on ensuring dignified supervision, documentation, and identification of dead. ${ }^{[3]}$ At present in COVID-19 pandemic, ICRC had been asked by different government to lay relevant practical guidelines in congruence with evidence-based experience this far in current epidemics. ${ }^{[3]}$ While planning or implementing management of dead with confirmed or suspicious COVID-19 status, safety and

${ }^{1}$ Assistant professor, Department of Anatomy, Ganesh Shankar Vidyarthi Memorial Medical College, Kanpur, Uttar Pradesh, India, ${ }^{2}$ Professor and Head, Department of Anatomy, Ganesh Shankar Vidyarthi Memorial Medical College, Kanpur, Uttar Pradesh, India

Address for correspondence:

Dr. Nidhi Gupta, Ganesh Shankar Vidyarthi Memorial Medical College, Kanpur, Uttar Pradesh, India. Postal Address: Quarter No. 21/ New Type 4. GSVM Medical College Campus, Kanpur - 208 002. Phone: +91-8318281017. E-mail: drnidhianat@gmail.com 
well-being of health personnel staff according to the best forensic practices need to be considered. ${ }^{[3]}$

Based on advisory of ICRC and advisory of Indian Council of Medical Research (ICMR), Government of India on March 18, 2020, declared that it is "unlikely to be an increased risk of infection from a dead body" of a coronavirus patient to health workers or family members who follow standard precautions, while issuing guidelines list for handling corpses. ${ }^{[4]} \mathrm{A}$ proposal of sensitization that novel coronavirus does not pose an additional risk was made to crematorium and burial ground staff. ${ }^{[4]}$ The guidelines stated that being a new disease, there is a knowledge gap on how to dispose of the dead body of a suspect or a confirmed case of COVID-19. This guideline was based on the existent epidemiological knowledge about the disease. However, no guidance was issued for recently acquired cadaver and personnel management specific to anatomy. Later, even in interim guidance dated March 24, 2020, the World Health Organization missed any word of instructions or advisory for anatomist across world. ${ }^{[5]}$ Looking at above picture, we need to analyze and confer the current pandemic from anatomist viewpoints of India and world.

In India, Medical Council of India (MCI) regulations for prerequisite of academic resources in morbid anatomy needs 25 cadavers for a strength of 250 students, mortuary cooler with arrangement to keep at least 8 bodies or suitable alternative arrangement, $1 / 2$ storage tank to hold 10 cadavers, made of concrete with copper lid, plastic tanks for storing soft and dissected parts, weighing machine for cadavers, and 2 embalming machines for cadavers. ${ }^{[6]}$ At present, there is huge need for supply of cadavers in various medical institutes due to mushrooming medical institutes. Usually, the most common source had been unclaimed destitute bodies obtained by police. ${ }^{[7]}$ In addition, non-government organizations also arrange cadavers donation for institutions. ${ }^{[7]}$ In India, in 1949, Anatomy Act was enforced and was then called as the Bombay Act (Bombay Anatomy Act, 2018). Subsequently, other states evolved their own Anatomy Acts and rulings. ${ }^{[7]}$ Previously, we had only problem of settling the erroneous/unethically labeled unclaimed bodies in coherence with the laws of state, now thanks to COVID-19 procurement will be even scarce and scary. Probably sooner or later, with introduction of virtual anatomy dissection rapidly overtaking wet cadaveric dissection particularly in epidemic time, new amendments and provisions are likely to be introduced. ${ }^{[7]}$ Hopefully, it will be compliant with more pragmatic and scientific "The Human Organs Transplantation Act”, hence, torch bearers of Anatomy will need to learn and update with the aspects of laws additionally. ${ }^{[7]}$ Since we need to scrutinize aspects of recent and upcoming orders, laws, and ethics for procuring cadaver in anatomical dissection, probably in post-pandemic changed anatomy wet dissection academic schedules, we need to revisit our strength and weakness in abiding with MCI norms. We have seriously missed evidence-based protocols on obtaining unclaimed destitute bodies for anatomy dissection from high-risk autopsy suite of postmortem department. So far, our only criterion had been whims and fancy of anatomy expert experiences in such selections. A consensus revised guidelines for optimization of resources needs to be established. In future medical history, records will be more relevant while accepting cadavers for official statistical and legal purpose. Ethical and humanitarian channels will be included with present administration deciding the whereabouts of cadavers.

We have so far worked on assumption that embalming process ameliorates the microbes which may pose variable risks in different parts of the world such as tuberculosis, streptococcal infection, gastrointestinal organisms, Creutzfeldt-Jakob disease, viral hepatitis, HIV infection, MERS, and other viral infections (particularly viral hemorrhagic fevers such as Lassa, Marburg, or Ebola), and possibly meningitis and septicemia (especially meningococcal) ${ }^{[8]}$ We certainly lack evidence for such hypothesis. COVID-19 had been an eye opener in making us realize that, we do not have serious protocols for embalming procedure, management of cadaver processing area, safety of mortuary personnel, and decontamination of instruments while handling biohazards.

Anatomy faculties are usually involved for undertaking embalming as a straight forward temporary preservation method. Majority of this procedure happens at anatomy department. ICMR proclaimed gradual decline of coronavirus from dead body while admitting that there is no specific duration to declare a body as non-infective, in response to a question of over how many days is the infectivity period of COVID-19 in a dead body..$^{[4]}$ The ICMR admitted considering the relatively high rate of false-negative results of COVID-19, every case still has to be considered as a possible coronavirus infection case, and hence, the new guidelines suggest that embalming of dead body should not be allowed and that autopsies should be avoided..$^{[4]}$ If an autopsy 
is to be performed for special reasons, the infection prevention control practices should be adopted. ${ }^{[4]}$ However, concerns over use of cadavers for academic purpose were neither asked nor answered. ${ }^{[4]}$ Having heard that, anatomists worldwide will be concerned with the question of how long is how much long. We believe in future, we will be considering every received body wrapped in impervious bag as potential hazard. To handle such situation ethically and with dignity for deceased body, we need to inculcate additional considerations in donning and doffing requisite currently in practice. ${ }^{[5]}$ Such deliberation will mitigate extra risk to embalmers. Embalming practices such as the open drainage of the vascular system lead to excessive environmental contamination and should be avoided. ${ }^{[5]}$ At present, there is empirical use of hypochlorite (e.g., chlorine bleaches) in medical establishment for decontamination. ${ }^{[5]}$ Hypochlorite is corrosive and may damage surfaces or instruments. Formaldehyde is likely to be present in postmortem rooms. Moreover, the reaction between hypochlorite and formaldehyde can produce a potent carcinogen (bischloromethyl ether). ${ }^{[8]}$ This indeed, is a very interesting observation missed by majority of anatomist. We need to arrive at consensus of using which disinfectants should be used to clean up any spills of blood or body fluid. We propose all instruments used for embalming should be carefully cleaned in detergent and either sterilized in an autoclave, boiled, or soaked in a disinfectant.

In this era of social alienation and mistrust bred from safe-distancing and doubtfulness, upcoming young students will be precarious of viewing the cadaver as theirs first patient. Unless told precisely about sanctity of cadaver and effective sanitization done beforehand, they might develop false notion of being exposed to infected cadaver ${ }^{[6]}$ They are quite likely to drift away from classical attraction of ageold anatomy dissection. In the current period of changed perceptions, we are likely to face variable issues and reactions for respect, compassion, smell, emotions, awareness of bereavement, body donations, teamwork skills, and orientation with anatomy subject itself in dissection hall. We need to work on student centric coping strategies and orientation. There are no dedicated institutional guidelines for wearing single-use gloves, impervious single-use aprons, and applicability of a filtering facemask giving protection equivalent to US NIOSH standard N95 or European standard FFP2. ${ }^{[8]}$ Before we start wet anatomy dissection practical classes again, we require framing instructions for our students, since they keep oscillating between highrisk clinical department to relatively low-risk preand para-clinical block in pursuance of curriculum. We need to assess the awareness of hand wash among students during and after the procedures and before eating, drinking, or smoking. ${ }^{[5]}$

\section{Conclusion}

In view of the rapid evolution of scientific evidences about COVID-19 pandemic, our current knowledge, attitude, awareness, and practices for academic utility of cadavers need amendment. Hopefully, it might come handy for decision makers in administration and academics. There was and will be no denying in age old fact "mortui vivos docebunt" (the dead shall teach the living), however, this time differently after current pandemic.

\section{References}

1. Available from: https://www.news.cgtn.com/news/2020-0216/Two-coronavirus-cadavers-dissected-for-medical-studyWhy-so-late--O8ybmJ1Jfy/index.html. [Last accessed on 2020 Jun 26].

2. Available from: https://www.buzzfeednews.com/article/ danvergano/coronavirus-spread-dead-body-coroner. [Last accessed on 2020 Jun 25].

3. Finegan O, Fonseca S, Guyomarc'h P, Morcillo Mendez MD, Rodriguez Gonzalez J, Tidball-Binz M, et al. International Committee of the Red Cross (ICRC): General guidance for the management of the dead related to COVID-19. Forensic Sci Int 2020;2:129-37.

4. Available from: https://www.indiatoday.in/india/story/ infection-unlikely-to-spread-from-dead-body-only-lungs-ofcovid-19-patients-pose-some-risk-govt-1656682-2020-03-18. [Last accessed on 2020 Jun 24].

5. Available from: https://www.apps.who.int/iris/bitstream/ handle/10665/331538/WHO-COVID-19-lPC_DBMgmt2020.1-eng.pdf. [Last accessed on 2020 Jun 25].

6. Available from: https://www.mciindia.org/CMS/wp-content/ uploads/2017/10/minimum-standard-requirements-for-100admissions.pdf. [Last accessed on 2020 Jun 25].

7. Pampilly VS. Cadavers for anatomical dissection. Indian $J$ Med Ethics 2005;2:16-7.

8. Availablefrom:https://www.isid.org/guide/infectionprevention/ humancadavers. [Last accessed on 2020 Jun 26].

\section{Financial Support: None; Conflicts of Interest: None}

How to cite this article: Gupta N, Pandey S. Learning, Unlearning, and Relearning Issues of Anatomy Cadaver Management during COVID-19 Pandemic in India and World: Thoughts for Present and Probable

Future Trends. J Med Sci Health 2020;6(2):63-65

Date of submission: 27-06-2020

Date of review: 05-07-2020

Date of acceptance: $18-08-2020$

Date of publication: 10-10-2020 